\title{
A INFLUÊNCIA DA VIBRAÇÃO MECÂNICA DA CARGA DE CARVÃO NOS ÍNDICES CSR E CRI DO COQUE METALÚRGICO
}

\author{
Felipe Inácio Cunha Alves ' \\ Leandro Miranda Nascimento ' \\ Guilherme Liziero Ruggio da Silva ' \\ Paulo Santos Assis '
}

\section{Resumo}

É indiscutível a necessidade da siderurgia de sempre se inovar e aprimorar seus métodos de produção para que as empresas mantenham sua competitividade no mercado. Dessa forma esse trabalho visa aperfeiçoar a produção do coque metalúrgico nas coquerias pela utilização de vibração mecânica da carga de carvão carregada, a fim de se possibilitar maior carregamento no forno da coqueria e também a utilização de cargas com blends mais pobres e, consequentemente, mais baratas. A análise científica foi conduzida em escala experimental em um forno de soleira de capacidade de $4,5 \mathrm{~kg}$, assim que o forno era carregado a vibração mecânica era produzida por um vibrador de imersão mecânico, esse comumente utilizado para adensamento de concretos em construção civil. O coque produzido apresentou aumento de $21 \%$ do valor de CSR e redução de $26 \%$ do CRI. O resultado obtido contradisse alguns resultados da literatura que informam que o CSR e CRI não sofrem influência com a densidade da carga carregada, apesar de que esses estudos a técnica de adensamento utilizada foi a de stamp charging.

Palavras-chave: Coque; Processo; Otimização; Siderurgia.

\section{THE MECHANICAL VIBRATION INFLUENCE ON COAL CHARGE TO CSR AND CRI OF METALLURGICAL COKE}

\begin{abstract}
Steelmaking companies must be always being looking for innovation and how to develop their production methods, because this is unquestionable very important to keep the company competitiveness on world's market. This research is about an improvement of metallurgical coke production on coke ovens by coal charge mechanical vibration, on this way will be possible a bigger oven loading and an utilization of more poor coal blends, and, consequently, cheaper. The scientific research has happened on experimental scale on a $4,5 \mathrm{~kg}$ capacity coke oven, The mechanical vibration was produced by a mechanical immersion vibrator, the one commonly used for concrete compacting on civil construction. The coke produced increased by $21 \%$ of the CSR value and a $26 \%$ reduction of the CRI. The results obtained contradicts some results of the literature that report that the CSR and CRI are not influenced by loaded coal density, although these studies the densification technique used was stamp charging.
\end{abstract}

Keywords: Coke; Process; Optimization; Steelmaking.

\section{INTRODUÇÃO}

A siderurgia no mundo alcança patamares cada vez mais desafiadores para produzir um aço com qualidade adequada para o seu fim comercial e com preço competitivo para concorrer no mercado externo. A China é responsável por aproximadamente $50 \%$ da produção de aço no mundo e os outros países produtores de aço estão perdendo cada vez mais espaço para o maior produtor mundial, que consegue entrar no mercado externo com uma proposta a custo bem inferior aos países concorrentes.

Em virtude de $95 \%$ da produção de ferro primário mundial ser realizada em altos-fornos a coque, observa-se uma grande oportunidade na área de produção [I]. Além disso, a produção de coque representa aproximadamente $40,5 \%$ dos custos de produção do ferro gusa, o que demonstra que

'Rede Temática em Engenharia de Materiais - REDEMAT, Universidade Federal de Ouro Preto - UFOP, Ouro Preto, MG, Brasil. E-mail: felipe.icalves@gmail.com 
a coqueria é uma área de bastante influência no preço final de produção do aço, influenciando diretamente na competitividade da empresa [2].

Nesse estudo busca-se avaliar a possibilidade de aplicação em escala industrial da vibração mecânica da carga da coqueria, a fim de se possibilitar um maior carregamento do forno. Além disso será avaliado o impacto da produção do coque de alta densidade nos parâmetros de qualidade de Coke Reactivity Index (CRI) e o Coke Strenght after Reaction (CSR), um impacto positivo possibilita a utilização de misturas mais pobres, e, consequentemente, uma redução de custos com blends de carvão somada ao aumento de produtividade mencionado.

Um coque com um alto $C R I$ indica que esse possui uma alta reatividade, porém deve se buscar um coque com baixo índice CRI, porque uma alta reatividade implica em uma alta reação com os gases da oxidação no alto-forno, implicando em um crescimento da proporção de $\mathrm{CO} 2$, assim o coque será enfraquecido e degradado implicando em redução da permeabilidade, queda de eficiência do alto-forno, aumento do consumo de coque e até mesmo bloqueio das ventaneiras do alto-forno com resíduos de coque [3]. Em relação ao CSR deve-se almejar um coque com alto CSR, isto implica em maior resistência, melhora a permeabilidade aos gases e líquidos, aumenta a produtividade do alto-forno e também diminui a taxa de consumo de coque [4].
Em 2005, Wright et al. [5] realizaram um estudo a respeito da influência da densidade sobre os valores de CSR e CRI. Nessa pesquisa eles concluíram que o coque que passa por um processo de adensamento apresenta os mesmos valores de CSR e CRI quando comparado ao coque que passa por um processo de produção normal. A justificativa dos resultados foi que os índices de CSR e CRI dependem primordialmente das propriedades da mistura de carvão.

O forno de soleira aquecida é um tipo de forno comumente utilizado para analisar a contração e dilatação do carvão e coque, esse forno possui várias diferenças em relação aos fornos industriais, temperatura, estrutura física e tempo de coqueificação, porém o coque produzido tem qualidades físicas, químicas, metalúrgicas e microestruturais semelhantes ao coque produzido em forno industrial, um exemplar desse forno pode ser observado na Figura I [6].

\section{MATERIAIS E MÉTODOS}

O coque produzido no forno de soleira aquecida é um produto de excelente qualidade de análise para este trabalho. $O$ forno de soleira utilizado possui as medidas de carregamento de $28 \mathrm{~cm} \times 28 \mathrm{~cm} \times 27 \mathrm{~cm}$ que pode ser carregado com até $4,5 \mathrm{~kg}$ de carvão para a produção de coque e esse forno pode ser observado na Figura 2.

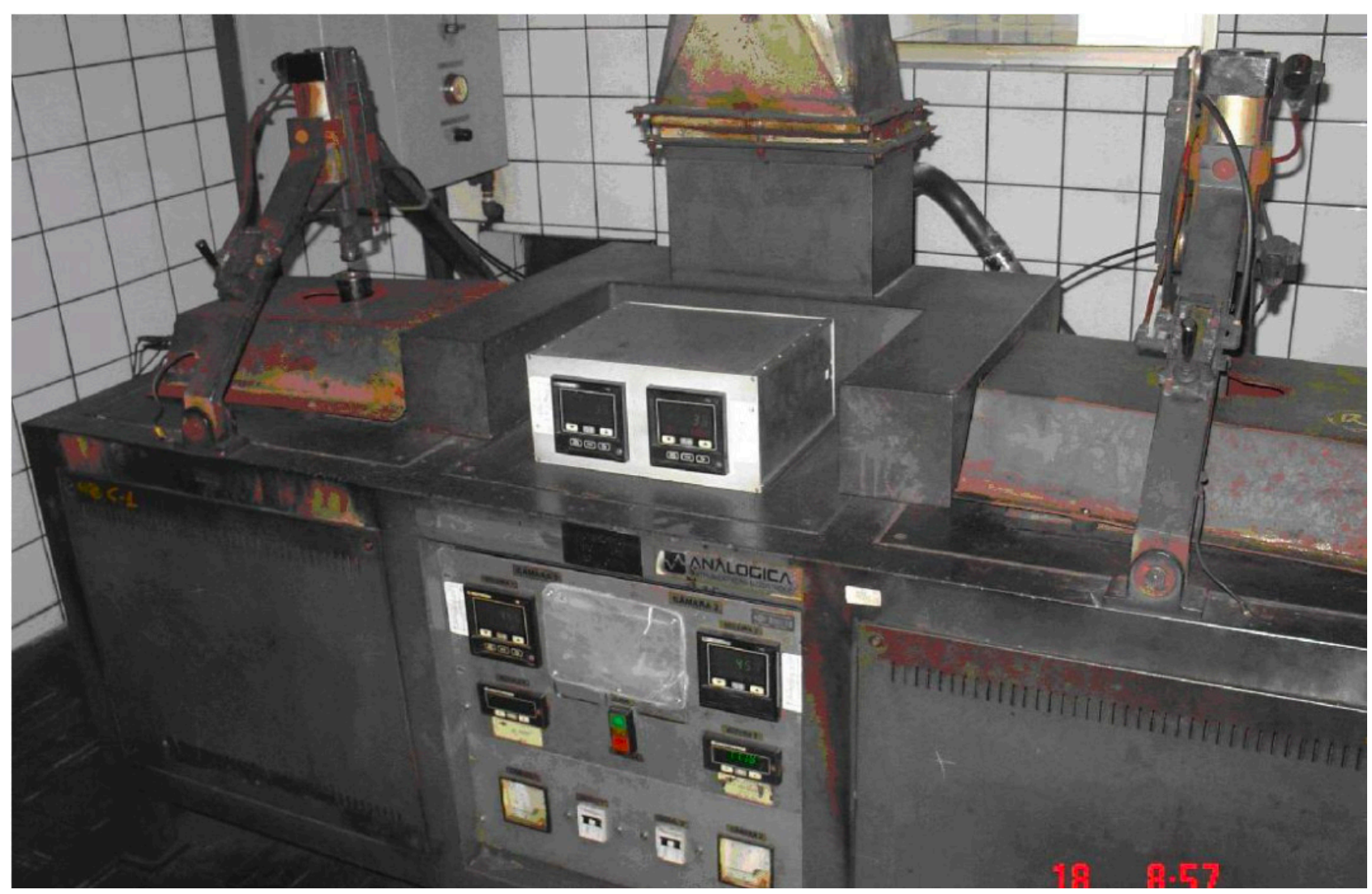

Figura I. Esquema de forno de soleira aquecida [6]. 
A composição química da mistura base de testes do carvão utilizado deve ser mantida em sigilo, então não será possível transcrever essa informação no trabalho em questão.

A vibração mecânica foi produzida por um vibrador de imersão portátil, comumente usado para adensamento de concreto na construção civil. $O$ modelo consiste em um motor acionado por gatilho com uma extremidade própria para o encaixe de uma mangueira flexível por um sistema combinado de encaixe, com encaixe macho-fêmea e rosqueamento. A mangueira flexível apresenta uma ponta metálica, no interior dessa ponta há um peso excêntrico que ao ser rotacionado vibra e transmite ondas de compressão e tração alternada para o sistema ao qual ele está imerso.
O modelo do vibrador usado pode ser observado na Figura 3 e as especificações do vibrador de imersão portátil estão explicitadas na Tabela I.

Tabela I. Especificações do vibrador de imersão portátil

\begin{tabular}{lc}
\hline \multicolumn{1}{c}{ Item } & Valor \\
\hline Potência $(\mathrm{W})$ & 1500 \\
Frequência do Motor $(\mathrm{Hz})$ & 60 \\
Rotação do Motor (RPM) & 18000 \\
Frequência de Oscilação $(\mathrm{vpm})$ & 12500 \\
Amplitude de vibração $(\mathrm{mm})$ & 8 \\
Diâmetro $(\mathrm{mm})$ & 28 \\
Comprimento Útil $(\mathrm{mm})$ & 385 \\
Comprimento Total $(\mathrm{m})$ & $\mathrm{I}, 5$ \\
\hline
\end{tabular}

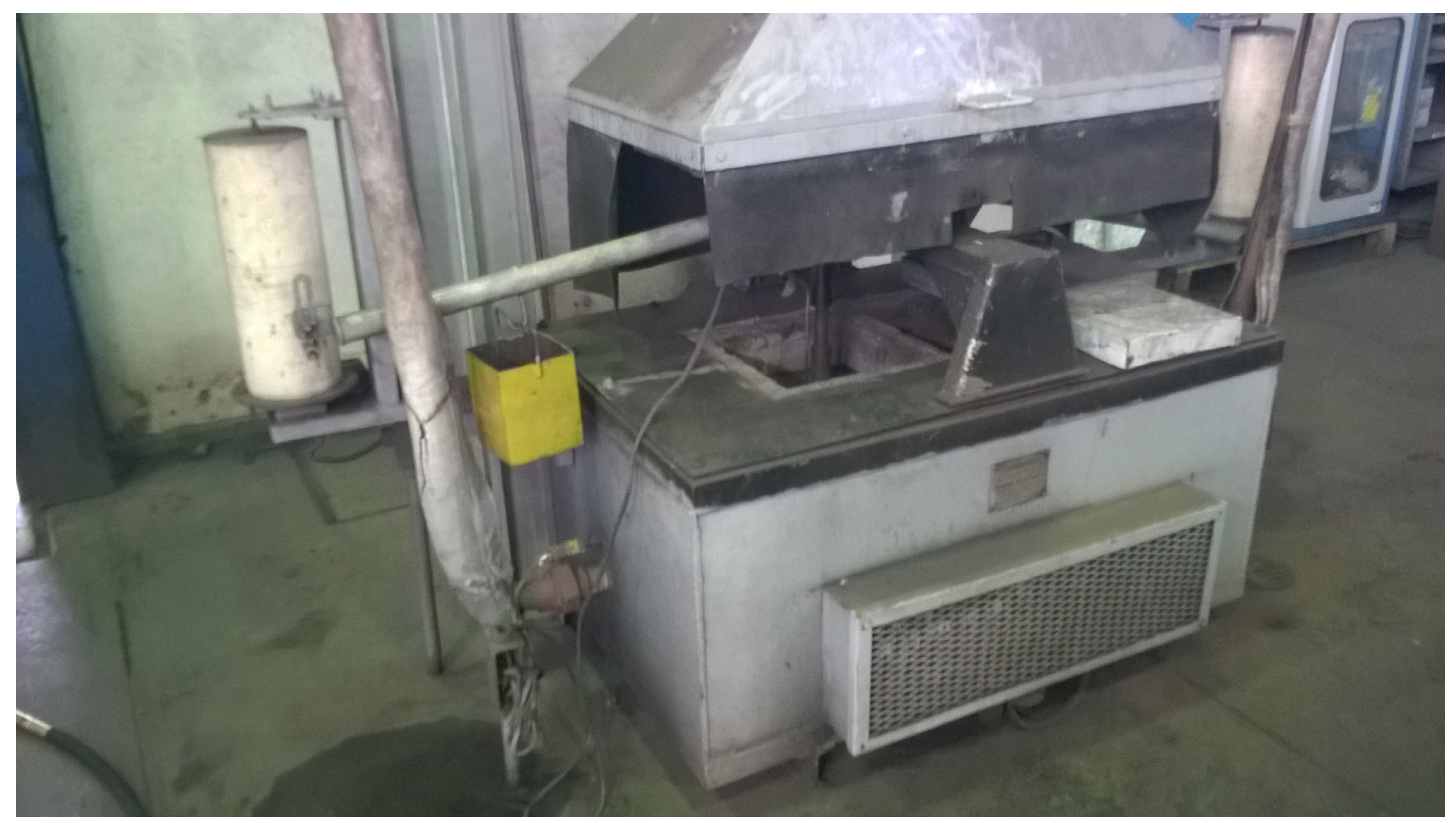

Figura 2. Forno de soleira aquecida.

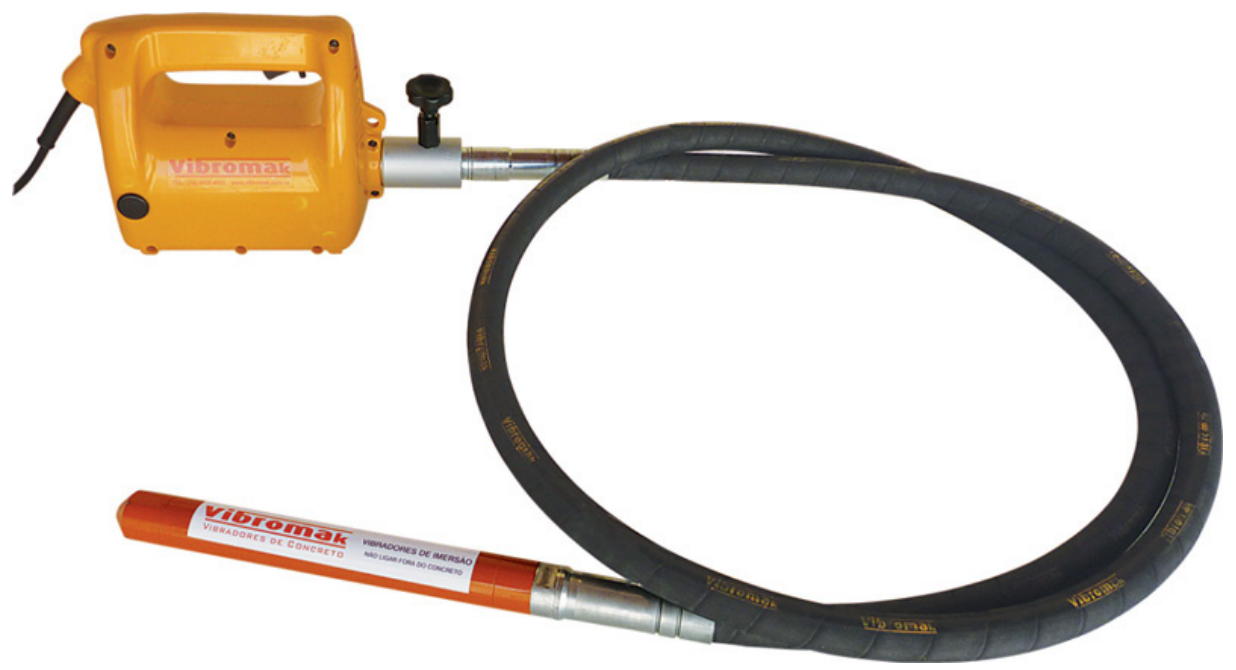

Figura 3. Vibrador de Imersão. 
A comparação entre as reatividades do coque CSR e CRI para as amostras densificadas e não densificadas será realizada segundo a norma ASTM 534I [7].

\section{RESULTADOS}

Foram produzidas amostras em duplicata de carvão vibrocompactado com $0 \%$ (padrão), $10 \%$ e $30 \%$ de compactação. A medida do adensamento acontecia conforme as partículas de carvão se ajeitavam ao volume do forno e a altura da amostra total diminuía, essa alteração na altura era acompanhada através de uma régua padronizada na parede interna do forno soleira. Desta forma já tinha-se a largura $(28 \mathrm{~cm})$, comprimento $(28 \mathrm{~cm})$, capacidade de carga do forno $(4500 \mathrm{~g})$ definidos.

A referência da altura da amostra padrão foi definida como $9 \mathrm{~cm}$, então à medida que o vibrador de imersão era inserido a altura das amostras teria que reduzir para $8,2 \mathrm{~cm}$ ( $10 \%$ adensamento), e $6,9 \mathrm{~cm}$ ( $30 \%$ de adensamento), conforme pode ser observado na Tabela 2.

Os resultados das análises de CSR e CRI médios das amostras obtidas em duplicatas podem ser observados na Tabela 3.

O índice de CSR, comparando o adensamento de $0 \%$ com o de $30 \%$, chegou a aumentar aproximadamente $21 \%$, melhorando consideravelmente resistência, permeabilidade, produtividade do forno e diminuindo consumo de coque. A curva de tendência do CSR pode ser observada na Figura 4.

Já para o índice de CRI obteve-se uma redução de aproximadamente $26 \%$, quando comparada a amostra de $10 \%$ e a de $30 \%$ de adensamento, outro resultado também almejado na área de produção de coque, pois o coque com baixo índice de reatividade implica em melhoras substanciais na resistência do coque, produtividade do forno e redução do consumo do mesmo. A curva de tendência para o CRI pode ser observada na Figura 5.

O aumento do CSR e redução do CRI contradiz os resultados obtidos por Wright et al. em 2005 [5], quando foi afirmado que os valores do CSR e CRI não variavam com a densidade da carga utilizada, pois esses índices eram propriedades da mistura do carvão. Provavelmente ocorreu porque o nível de adensamento em seu estudo foi baixo, já que entre 0 e $10 \%$ de adensamento o valor de CSR praticamente não se alterou. $\mathrm{Na}$ atual pesquisa obteve-se o resultado que um alto aumento de densidade da carga, 30\% nesse caso, melhora os parâmetros físicos do coque obtido.

Para realizar uma análise de produtividade de retorno manteve-se todos os parâmetros tradicionais de uma coqueria, só mudando a massa carregada para a análise de produtividade, esse ganho que pode ser observado na Tabela 4. Supondo um forno tradicional de coqueria que tenha capacidade de 22 toneladas e a capacidade total anual de produção de de 1.600 .000 toneladas. Carregando-se com uma carga de carvão $30 \%$ adensada, será possível aumentar a capacidade de carga e obter mais coque a partir desse forno, que é exatamente os mesmos $30 \%$ de ganho de massa, implicando em um aumento de $30 \%$ de
Tabela 2. Indicação para padronização das amostras de carvão inseridas no forno

\begin{tabular}{|c|c|c|c|c|c|c|}
\hline 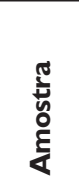 & 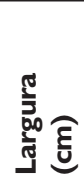 & 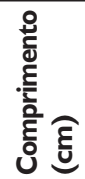 & 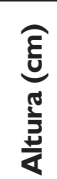 & 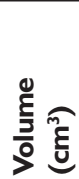 & 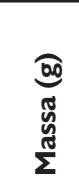 & 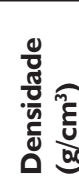 \\
\hline $0 \%$ & 28 & 28 & 9 & 7056 & 4500 & 0,638 \\
\hline $10 \%$ & 28 & 28 & 8,2 & 6415 & 4500 & 0,702 \\
\hline $30 \%$ & 28 & 28 & 6,9 & 5428 & 4500 & 0,829 \\
\hline
\end{tabular}

Tabela 3. Resultados médios de CSR e CRI

\begin{tabular}{ccc}
\hline Amostra & CSR & CRI \\
\hline 0 & 49,86 & 32,89 \\
10 & 48,48 & 31,11 \\
30 & 60,34 & 24,06 \\
\hline
\end{tabular}

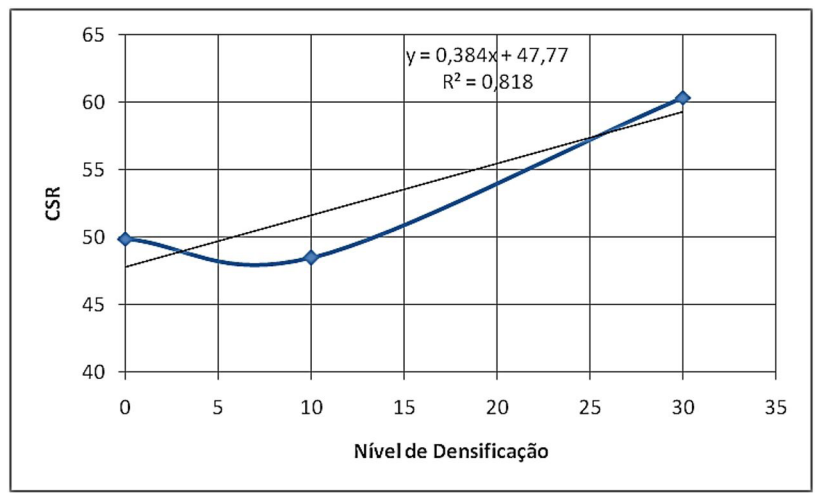

Figura 4. Curva de tendência para o CSR.

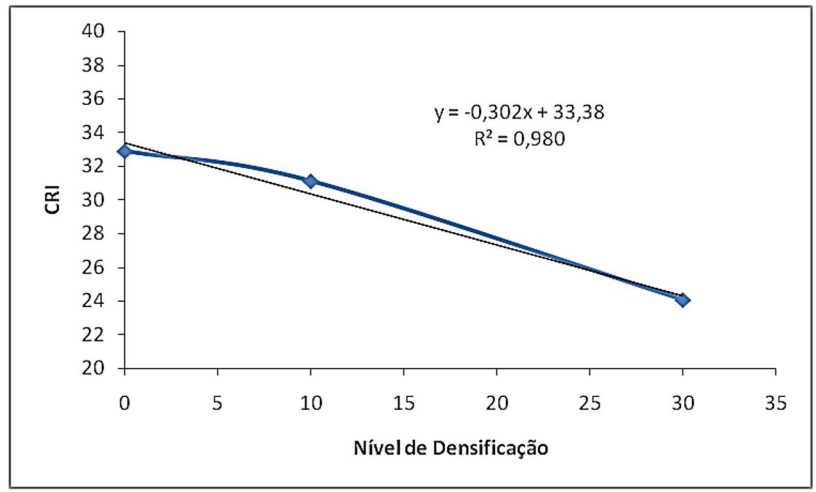

Figura 5. Curva de tendência para o CRI.

Tabela 4. Análise do aumento de produtividade

\begin{tabular}{lc}
\hline Capacidade do Forno c/ Mistura Padrão (ton) & 22 \\
Capacidade do Forno c/ Mistura 30\% Adensada (ton) & 28,6 \\
Aumento de Produtividade por Forno (ton) & $\mathbf{6 , 6}$ \\
Capacidade de Produção da Coqueria (ton/ano) & 1600000 \\
Capacidade de Produção da Coqueria 30\% (ton/ano) & 2080000 \\
Aumento de Produtividade (ton/ano) & $\mathbf{4 8 0 0 0 0}$ \\
\hline
\end{tabular}


produtividade, desconsiderando-se qualquer mudança nos parâmetros da coqueria em função do maior volume de carga. Desta forma a coqueria em questão seria capaz de produzir 480.000 toneladas a mais do que as I.600.000 toneladas já produzidas anualmente.

\section{CONCLUSÃO}

Os resultados obtidos com a utilização de carga adensada foram completamente favoráveis aos parâmetros de produção do coque, implicando em um coque de alta qualidade e aumento de produtividade da coqueria.

O índice de CSR para a carga adensada aumenta cerca de $21 \%$ em função da vibração mecânica da carga, sendo que de 0 a $10 \%$ praticamente não se alterou o índice. O índice de CRI diminui aproximadamente $26 \%$ em função do aumento de densidade da carga de 0 a $30 \%$.

O aumento de produtividade obtido é plausível, observando que uma carga de maior densidade ocupa menor volume, assim a capacidade de carga dos fornos é aumentada e, consequentemente, a produtividade.

\section{Agradecimentos}

Os autores agradecem à GERDAU por todo suporte técnico e estrutura, CAPES pelo financiamento e à REDEMAT por toda a base de conhecimento fornecida.

\section{REFERÊNCIAS}

I Osório E, Vilela ACF, Sampaio CH. Estudo prospectivo do setor siderúrgico. Brasília: Centro de Gestão e Estudos Estratégicos; 2008.

2 Coelho RJ, Silva OJ, Alves MT, Andrade LA, Assis PS. Modelos de previsão da qualidade metalúrgica do coque a partir da qualidade dos carvões individuais e do coque obtido no forno-piloto de coqueificação. Revista Escola de Minas, 2004;57(I):27-32.

3 Díez RMA, Alvarez CB. Coal for metallurgical coke production: predictions of coke quality and future requirements for cokemaking. International Journal of Coal Geology. 2002;50(I-4):389-4I2.

4 Hilding T, Kazuberns K, Gupta S, Sahajwalla V, Sakurovs R, Björkman B, et al. Effect of temperature on coke properties and $\mathrm{CO}_{2}$ reactivity under laboratory conditions and in an experimental blast furance. In: AISTech 2005 Proceedings; 2005; Charllote. Warrendale, PA: Association for Iron \& Steel Technology's; 2005.

5 Wright R, Kim R, Schücker FJ. Compacting of coal for heat recovery ovens. In: Proceedings of the 5th European Iron \& Cokemaking Conference; 2005. Stockolm. Stockolm: ASMET: The Austrian Society of Metallurgy and Materials; 2005.

6 Casagrande MC. Avaliação dos parâmetros de CSR e CRI do coque produzido em forno industrial, forno soleira aquecida e box test. Ouro Preto: REDEMAT; 2010.

7 American Society for Testing and Materials. ASTM 534I: Standard Test Method for Measuring Coke Reactivity Index (CRI) and Coke Strength After Reaction (CSR). West Conshohocken: ASTM.

Recebido em: 16 Jan. 2017

Aceito em: 26 Abr. 2017 